\title{
Journal of Methods and Measurement
}

\section{Reviewers}

Lee Sechrest, University of Arizona, AZ, USA

Ghadah Alkhadim, Taif University, KSA

Kevin Bird, University of New South Wales, Sydney, Australia

Tomas Cabeza de Baca, University of Arizona, USA

Candace Black, University of Arizona, AZ, USA

Tom Booth, University of Edinburgh, Edinburgh, Scotland

Richard Bootzin, University of Arizona, AZ, USA

Bethany Bray, Pennsylvania State University, PA, USA

Sacha Brown, University of Arizona, AZ, USA

M. H. Clark, University of Central Florida, FL, USA

Amy Tsang Davis, University of Arizona, AZ, USA

Katherine Cummings Davis, Center for Biological Diversity, AZ, USA

Cameron Davis, Arizona State University, AZ, USA

Owen Davis, University of Arizona, AZ, USA

Vajeera Dorabawila, Office of Children and Family Services, NY, USA

Aurelio José Figueredo, University of Arizona, AZ, USA

David Furrow, Mount Saint Vincent University, Halifax, NS, Canada

Jonathan Gable, University of Arizona, AZ, USA

Rafael Garcia, University of Arizona, AZ, USA

Eric Heckler, Arizona State University, AZ, USA

Dwight M. Hite, Cameron University, OK, USA

Kathleen Insel, University of Arizona, AZ, USA

W. Jake Jacobs, University of Arizona, AZ, USA

James King, University of Arizona, AZ, USA

Beatrice Krauss, Hunter College, City University of New York, USA

Herbert H. Krauss, Pace University, NY \&

Hunter College, City University of New York, USA

Karen Larwin, Youngstown State University, USA

Frederic Malter, Max Planck Institute, Munich, Germany

Katherine McKnight, RTI International, OR, USA

Patrick McKnight, George Mason University, VA, USA

J. Michael Menke, International Medical University, Malaysia

Alan Neaigus, Columbia, NY, USA 
Félix Neto, University of Porto, Portugal

Sally Gayle Olderbak, Ulm University, Germany

Kimberly Phillips, University of New Hampshire, NH, USA

Beth Poindexter, Genesis Natural Medicine Center, Tucson, AZ, USA

Joshua R. Polanin, Vanderbilt University, Nashville, TN

Mark Roberts, British Columbia, Canada

Kenneth D. Royal, North Carolina State University, NC, USA

Mary V. Schaffer, Tucson, AZ, USA

Souraya Sidani, University of Ryerson, Toronto, Canada

David L. Streiner McMaster University Clinical, Hamilton, ON, CA

Alexander Von Eye, Michigan State University, East Lansing, MI, USA

Alex Weiss, University of Edinburgh, Scotland

David Weiss, University of Minnesota, MN, USA

David B. Wilson, George Mason University, VA, USA

Pedro S. A. Wolf, University of Capetown, South Africa

Kirsten Yaffe, University of Arizona, AZ, USA

William Yeaton, Tallahassee, FL, USA

Guili Zhang, East Carolina University, NC, USA 